\begin{tabular}{|c|c|c|}
\hline Beitr. Ent. & Keltern & ISSN 0005-805X \\
\hline $\mathbf{5 8}(2008) 2$ & S. $249-266$ & 15.11 .2008 \\
\hline
\end{tabular}

\title{
Die Anomaloninae der Sammlung Arnold FörSTER - Typenrevision und faunistische Anmerkungen
}

\section{(Hymenoptera, Ichneumonidae)}

\author{
Heinz SchneE
}

\section{Summary}

The 22 species of the subfamily Anomaloninae described by FörSTER are revised. Only the following five species have proved to be valid: Barylypa propugnator (FörSTER, 1855), Barylypa mesozona (FöRSTER, 1878), Erigorgus melanops (FöRSTER, 1855), Erigorgus foersteri (MoCsÁRY, 1897) (a replacement name for Anomalon brevicorne FöRSTER, 1855) and Atrometus insignis (Förster, 1878). Lectotypes are designated for seven species. 16 new synonymies are established. In the remaining FörSTER collection 41 species were found. They are briefly annotated faunistically and in view of their host relationships.

\section{Keywords}

FöRSTER collection, Anomaloninae, type revision, faunistics.

\section{Zusammenfassung}

Die 22 von Förster beschriebenen Anomaloninae werden überwiegend an Hand der erhaltenen Typen revidiert. Dabei zeigte sich, dass nur folgende fünf Arten valide Taxa sind: Barylypa propugnator (FöRSTER, 1855), Barylypa mesozona (FöRSTER, 1878), Erigorgus melanops (FöRSTER, 1855), Erigorgus foersteri (Mocsáry, 1897) (nom. nov. pro Anomalon brevicorne FöRsTer, 1855) und Atrometus insignis (Förster, 1878). Für sieben Arten werden Lectotypen festgelegt. Es ergaben sich 16 neue Synonyme. In der restlichen FörSTER-Sammlung fanden sich insgesamt 41 zu den Anomaloninae gehörende Arten, die kurz faunistisch und hinsichtlich ihrer Wirtsbindung kommentiert werden.

\section{Schlagwörter}

Sammlung Förster, Anomaloninae, Typenrevision, Faunistik.

\section{Einleitung}

Der bedeutende Hymenopterologe Arnold Förster (1810-1884) hat in drei Arbeiten (1855, 1860 und 1878) eine Reihe zur Unterfamilie Anomaloninae gehörender Arten beschrieben, die seitdem in der Mehrzahl nicht oder fehlerhaft gedeutet worden sind. Während die Interpretation der 1855 und 1878 publizierten Arten dank der ausführlichen Beschreibungen auch bei verschollenen Typen möglich ist, können die 1860 beschriebenen meist nur an Hand der Typen gedeutet werden.

Bekanntlich hat Förster (1869) im Rahmen einer Bestimmungstabelle 489 neue Ichneumoniden-Gattungen aufgestellt, ohne ihnen Arten zuzuordnen. Das gilt auch für fünf zu den 
Anomaloninae gehörende Genera. In fünf weiteren Gattungen wurden von ihm selbst 1860 und 1878 Anomaloninae-Arten eingeschlossen. Spätere Autoren, beginnend mit Brischke (1880) bis Perkins (1962), haben den erstgenannten fünf Gattungen Arten zugeordnet und für alle zehn die Gattungstypen festgelegt.

Aus nachgelassenen Tagebüchern FörSTERs geht hervor, dass er sowohl für die von ihm selbst als auch von anderen Autoren aufgestellten Anomaloninen-Gattungen typische Arten ausgewählt hat. Die im „Notizbuch Nr. V (angefangen im Jahre 1843?)“ zu findende Übersichtstabelle über die Gattungen der „Anomaloidae“ wurde 1869 fast unverändert in Form eines dichotomen Bestimmungsschlüssels übernommen. Warum er die Genotypen in den genannten Arbeiten über Anomaloninae nicht publiziert hat, ist unverständlich. Wegen der unzureichenden Charakterisierungen seiner Genera überrascht es nicht, dass einige der nach Artikel 69.1. ICZN (2000) nicht mehr revidierbaren Festlegungen späterer Autoren nicht im Sinne FörsTERs erfolgt sind.

Schmiedeknecht war im Besitz der Tagebücher, aus denen er 1888 in der Bearbeitung der Pimplinae zitiert (Horstmann 1990). Leider wurden von ihm die darin enthaltenen Informationen über die Gattungstypen Försters in seiner Monographie der Anomaloninen (1902, 1903) und auch später $(1935,1936)$ nicht genutzt.

Da der größte Teil der Anomaloninen-Sammlung FörsTers erhalten geblieben ist, war es möglich, dieses interessante Material zu sichten und einen Gesamtüberblick über seine Auffassung von der Unterfamilie zu erhalten. Dabei zeigte sich einmal mehr, dass er die Artgrenzen viel zu eng zog, vornehmlich auf der Basis mitunter geringfügiger koloristischer Unterschiede.

\section{Zur Aufbewahrung und Etikettierung der Typen}

FöRSTER hat die 1855 beschriebenen neuen Hymenopteren nach einer Mitteilung von MocsáRY (1897, 1898) im Jahre 1851 von J. von FrIVALDSZKY, einem ungarischen Entomologen, zur Bestimmung erhalten. Daraus lässt sich schließen, dass sie nach der Bearbeitung wieder zurückgeschickt worden sind. Försters eigene Angaben zur Herkunft der neuen Arten lassen keine eindeutige Schlussfolgerung auf den Verbleib des Typenmaterials zu. Bei einer Reihe von Arten verwendet er die Formulierung "Von FrIVALDSZKY aus Ungarn erhalten", bei den vier Anomaloninen dagegen lediglich "Aus Ungarn". Nach Mocsáry (1898) befindet sich die von von FrivaldszKY zusammengetragene Sammlung im Magyar Természettudományi Múzeum Budapest (HNHM). Eine Arbeit MocsÁrys von 1879 enthält Fundortangaben, u. a. auch für die vier Förster-Typen von 1855. Diese Daten kann er nur von von FrIVALDSZKY selbst erhalten oder einem jetzt nicht mehr vorhandenen Katalog entnommen haben, denn Fundortetiketten fehlen völlig. Anlässlich eines Besuches in Budapest konnte ich drei Typen finden. Die Etikettierung besteht aus je einem Nummernetikett und bei zwei Typen außerdem aus Bodenetiketten mit den Artnamen, wahrscheinlich von Mocsárys Hand. Determinationsetiketten von FörsTER selbst fehlen.

Die Zoologische Staatssammlung München (ZSM) beherbergt einige wenige Anomaloninae aus der Förster-Sammlung ohne Typusstatus. Der größte Teil der Typen der 1860 und 1878 beschriebenen Arten sowie weiteres umfangreiches Material befinden sich im Zoologischen Museum Berlin (ZMHB). Sie sind in die Hauptsammlung des Museums eingeordnet worden. Dabei erhielten alle Exemplare die Kennzeichnung „ex coll. Förster“. Bei den meisten Anomaloninae weist das erste Exemplar einer Serie ein großes, blau umrandetes, kalligraphisch geschriebenes Etikett mit dem Artnamen auf (siehe Bachmaier 1979, Abb. 1). Ganz vereinzelt finden sich auch kleine Determinationsetiketten mit Försters normaler Handschrift. In den meisten Fällen stecken Nummernetiketten unter den Tieren. Die Bedeutung der Nummern in Bruchform konnte ich nicht ermitteln. Wahrscheinlich handelt es sich um Katalog-Nummern, doch sind 
weder in Berlin noch in München (DiLler, 1993) Kataloge von Förster vorhanden. Auch in den erhalten gebliebenen Notizbüchern fehlt jeglicher Bezug auf die Nummern. Eine Reihe von Exemplaren ist mit Fundortetiketten versehen. Es handelt sich dabei einmal um gedruckte, z. B. "Aachen Juni", zum anderen um kleine, häufig mit roter Tinte beschriftete Etiketten, z. B. "Köln" (s. Bachmaier 1979, Abb. 1).

Der Erhaltungszustand der Typen ist vor allem als Folge von Anthrenenfraß nicht besonders gut. Das gilt auch für viele Tiere in der sonstigen Anomaloninen-Sammlung Försters. Wenn das Geschlecht fraßbedingt nicht mehr zu erkennen war, steht hinter der Anzahl der vorhandenen Tiere die Abkürzung Ex. (für Exemplare).

\section{Typenrevision}

Während Förster die 1855 beschriebenen Arten noch bei Anomalon sensu Gravenhorst einordnete, stellte er 1860 die neuen Gattungen Habronyx und Agrypon auf. Habronyx gründete er vor allem auf die deutlich gekämmten Fußklauen. Als charakteristisch für Agrypon wertete er den nicht gebrochenen Nervellus und stellte in einer Bestimmungstabelle 12 neue Arten sowie zwei tatsächlich und eine vermeintlich von Gravenhorst beschriebene Species dazu. Die Tabelle basiert überwiegend auf Farbmerkmalen und auf der Flügeladerung, wobei FöRSTER, wie schon erwähnt, die Variabilität der Färbung völlig unterschätzte. Dementsprechend sind die von ihm 1860 beschriebenen Anomaloninae, soweit sie gedeutet werden konnten, Synonyme. SzÉPLIGETIS (1905a) partielle Deutungsversuche erwiesen sich ohne Kenntnis der Typen als falsch.

In seine 1869 begründete und 1878 ausführlicher definierte Gattung Laphyctes hat Förster die Arten L. mesozonus, L. insidiator und L. uniguttatus (GravenHorst) gestellt. (Laphyctes Förster, 1869 ist ein jüngeres Homonym von Laphyctes Dujardin, 1844, Laphyctes Reichenbach, 1850 und Laphyctes STÅL, 1853). Als Unterschiede zu Barylypa Förster nannte er den spitz vorgezogenen Clypeuszahn und den in oder über der Mitte gebrochenen Nervellus. Dabei unterlief ihm der Lapsus, die Art uniguttatus, die die Merkmale von Barylypa Förster 1869 aufweist, mit zu Laphyctes zu stellen. Daraus resultiert auch die Angabe bei Laphyctes, dass die Fühler beim Weibchen kurz, beim Männchen länger als der halbe Körper seien. Die kurzen Fühler kommen nur uniguttatus zu, während sie bei den zwei anderen Arten (in beiden Geschlechtern) deutlich länger als der halbe Körper sind.

FöRSTERS „Laphyctes uniguttatus m.“ (1878, S. 75) ist nicht als jüngeres sekundäres Homonym von Anomalon uniguttatum Gravenhorst aufzufassen. Der Autor meinte mit dem „m“ (= mihi) mit Sicherheit die neue Kombination von Gattungs- und Artnamen, denn er zitiert sofort die Art Gravenhorsts und er redeskribiert sie lediglich. Die beiden von Förster getrennten Taxa werden heute im Genus Barylypa zusammengefasst.

\section{Anomalon vicinum FöRSTER 1855, S. 232}

Holotypus ( $\sigma^{x}$ ) verschollen.

Locus typicus: Ungarn.

Gültiger Name: ? Barylypa rufa (Holmgren, 1857) (= ? B. vicina Förster).

Nach Mocsáry (1879) stammte der Holotypus aus Budapest. Szépligeti (1905b) ordnete die Art zu Unrecht in die Gattung Anomalon auct. (= Erigorgus Förster) ein, weil bei ihr nach Försters Angabe ebenso wie bei der folgenden Art der Postnervulus über der Mitte gebrochen ist. Damit ist auch die Synonymisierung mit Erigorgus latro (Schrank) durch SzÉpligeti und (als var. vicinum) durch Kiss von Zilah (1929) und Schmiedeknecht (1936) unzutreffend. 
Die Beschreibung FöRSTERs passt recht gut auf die als Barylypa rufa bekannte, vor allem in der Färbung außerordentlich variable Art. Allerdings ist der Nervus recurrens bei ihr meist interstitial, während er dem Autor zufolge beim Holotypus postfurcal liegt. Deshalb bleibt die Deutung unsicher, obwohl eine leicht postfurcale Lage des genannten Flügelnervs unter Dutzenden von Exemplaren von B. rufa hin und wieder vorkommt.

\section{Anomalon propugnator FöRSTER, 1855, S. 233}

1 ㅇ: Holotypus: "95", "A. propugnator" (Bodenetikett von MocsáRY?) (HNHM).

Locus typicus: Ungarn.

Anomalon rufum HabermeHL ,1920, S. 329.

1 ㅇ: Lectotypus (hiermit festgelegt): „Hinterz. Juli 17 Hab.“ „Syntypus Anomalon rufum HAB. SMF H2266a" (SMF).

1 : Paralectotypus: „Hinterz. Juli 17 Hab.“, „Syntypus Anomalon rufum HAB. SMF H2266b“ (SMF).

Locus typicus: Hinterzarten (Schwarzwald).

Gültiger Name: Barylypa propugnator (FörsteR, 1855) = Laphyctes insidiator FöRSTER, 1878 syn. n. = Anomalon carinatum Brischke, 1880 syn. n. = Anomalon rufum Habermehl, 1920 syn. nov. (primäres Homonym von Anomalon rufum Holmgren, 1857 und Anomalon rufum Provancher, 1874).

Der Arbeit von MocsáRy (1879) ist zu entnehmen, dass der Typus von Anomalon propugnator Ende Juni in Budapest (Miksavölgy) gefangen wurde. Die Autoren, beginnend mit SzÉPLIGETI (1905a, b) bis zu Atanasov (1975) haben die Art in die Gattung Anomalon sensu Gravenhorst bzw. Erigorgus Förster gestellt. Dabei ließen sie jedoch die eindeutige Angabe Försters außer acht, dass "die zweite Discoidalzelle an der Basis stark verengt", oder anders ausgedrückt, der Postnervulus weit über der Mitte gebrochen ist. Bei der Gattung Erigorgus ist dieser Nerv dagegen stets unter der Mitte gebrochen.

Die obigen Synonyme ergeben sich durch Vergleiche der Typen bzw. (bei Anomalon carinatum) aus der Originalbeschreibung.

Habermehls (1920) Anomalon rufum ist die relativ seltene weitgehend rot gefärbte Form von Barylypa propugnator.

\section{Anomalon melanops FörSTER, 1855, S. 234}

1 ㅇ: Holotypus: "99", "Erigorgus flavimanus Szépl. det. Szépl.", "Gravenhorstia melanobata melanops Först. det. Móczár" (HNHM).

Locus typicus: Ungarn.

Gültiger Name: Erigorgus melanops (FörSTER, 1855).

Der Holotypus stammt nach MocsáRY (1879) aus der Budapester Gegend (Farkasvölgy). Das Tier flog Ende April.

Im Gegensatz zur vorigen und zur nachfolgenden Art fehlt bei melanops ein Etikett (von MocsÁrY?) mit dem originalen Artnamen. Aber die Beschaffenheit des Nummernetiketts gleicht den entsprechenden Etiketten jener Arten völlig.

Das Exemplar entspricht der Beschreibung FörSTERs genau. Unter anderem erwähnt der Autor zwei individuelle Besonderheiten, die das Weibchen tatsächlich aufweist: 
1. "... das erste Fühlerglied auf der Unterseite mit einem kleinen roten Fleckchen."

2. "... die rücklaufende Ader in dem einen Flügel fast ganz als Fortsetzung der Cubitalquerader zu betrachten, in dem anderen Flügel dagegen etwas deutlicher davon getrennt."

Aus diesen Gründen bin ich sicher, dass das oben genannte Exemplar der Holotypus ist.

Bei E. melanops handelt es sich um eine gute Art, mit bei den Männchen stark, bei den Weibchen nur wenig variierender Gesichtsfärbung. Das konnte durch eigene Zuchtversuche an natürlichen, aber auch an experimentellen Noctuiden-Wirten nachgewiesen werden. Selbst bei den Nachkommen eines Weibchens war diese unterschiedliche Variabilität der Geschlechter deutlich ausgeprägt. Bei den Männchen variiert die Gesichtfärbung von ganz gelb bis weitgehend schwarz mit gelben Orbiten und einem mehr oder weniger großen gelben Mittelstreifen (unpubliziert). Diese Variabilität veranlasste einige Autoren, z. B. SzÉPligeti (1899), Brauns (1895) und auch FÖRSTER (in litteris) zu Neubeschreibungen von "Arten".

\section{Anomalon brevicorne FöRSTER, 1855, S. 236}

1 우 : Holotypus: „107”, „A. försteri" (Bodenetikett von MocsÁry?) (HNHM). Locus typicus: Ungarn.

Gültiger Name: Erigorgus foersteri (MOCSÁRY, 1897), nom. n. pro Anomalon brevicorne FörSTER, (= Anomalon försteri Dalla Torre, 1900, jüngeres Homonym), (= E. melanops auct.).

Nach Mocsáry (1879) wurde der Holotypus Mitte April im Raum Budapest (Sashegy) gefangen. SzÉpligeti (1905b) hat übersehen, dass sein Landsmann MocsáRY (1897) der Erste war, der den durch Gravenhorst präokkupierten Namen Anomalon brevicorne für Försters Art durch A. försteri ersetzte und deshalb das Homonym von Dalla Torre als gültigen Namen angenommen. Diese kleinste westpaläarktische Erigorgus-Art ist mit Ausnahme von SzÉPLigeTI von den Autoren nach Mocsáry (1879) verkannt worden. Sie stellten sie in die Synonymie von E. melanops (FÖRSTER).

\section{Habronyx Gravenhorstii FöRSTER, 1860, S. 148}

$10^{\star}$ : Lectotypus (von Townes et al. 1965 festgelegt): „Türkei“, „20|757“ ”, „Gravenhorstii Foerst.“, „ex coll. Förster“ (ZMHB).

1 올 Paralectotypus: „Türkei“, „20|757“ (ZMHB).

Locus typicus: Türkei.

Gültiger Name: Habronyx heros (WeSMAel, 1849).

Förster glaubte, seine Art auf Grund unterschiedlicher Größe und des auf Südeuropa beschränkten Wirtes von $H$. heros abgrenzen zu können. Er erhielt sie von von FrIvaldszKK, der sie aus der Lasiocampide Pachypasa otus (Drury) gezüchtet hatte. Diese Wirtsangabe wurde seitdem mehrfach bestätigt, zuerst durch Kriechbaumer (1880). Die Synonymie mit H. heros hat SzÉPLIGETI (1905a) zuerst erkannt. FörSTER erwähnt in seiner Beschreibung keinen Fundort. Aber einer Mitteilung von Mocsáry (1898) zufolge hat ihm von FrIVALDSZKY auch Tiere aus der Türkei geschickt, so dass der Typusstatus der beiden von Townes designierten Exemplare nicht zu bezweifeln ist.

\footnotetext{
${ }^{1}$ Die Nummernetiketten in Bruchform, z. B. „20“ werden in dieser Arbeit als „20|757“ dargestellt.
} 


\section{Trichomma ruficoxis FöRSTER, 1860, S. 149}

1 : Holotypus: „17|626“, „Aachen“, „Frst“, „,ruficoxis/Rossi/Foerst.“ (ZMHB).

Locus typicus: Aachen.

Gültiger Name: Trichomma enecator (Rossı, 1790).

Die Synonymisierung mit T. enecator geht auf SzÉPLIGETI (1905a, b) zurück. FöRSTER war zwar der Meinung, enecator unterscheide sich besonders in der Färbung wesentlich von ruficoxis, doch schon seine Beschreibung deutete auf Konspezifität hin. Der Holotypus bestätigt diese Annahme. Während die Artdiagnosen bei den beiden zuletzt genannten Arten recht ausführlich sind, lassen sie in der Tabelle für die Arten der neuen Gattung Agrypon Förster sehr zu wünschen übrig.

\section{Agrypon subclavatum FöRSTER, 1860, S. 151}

Typus verloren: Nadel ohne Exemplar: „21|573“, „Frst“, „subclavatum Foerst*“ (ZMHB). Gültiger Name: Agrypon flexorium (Thunberg, 1822) (= Agrypon subclavatum FörsTer, syn. nov.). Die Deutung kann sich hier nur auf die spärliche Diagnose in Försters Tabelle stützen. Die angegebenen Merkmale fügen sich in die große Variationsbreite von A. flexorium ein.

\section{Agrypon clandestinum FörSTER, 1860, S. 151}

Typen verschollen.

Gültiger Name: Agrypon clandestimum (Gravenhorst, 1829) (= A. clandestinum Förster, syn. nov.) (= jüngeres sekundäres Homonym).

Normalerweise ist der Nervellus bei dieser Art mehr oder weniger deutlich gebrochen. Es gibt aber Exemplare, bei denen die Brechung nur angedeutet ist.

Die von Förster in der Bestimmungstabelle verwendeten Merkmale sind zwar wenig präzise, sprechen aber nicht gegen die hier vorgenommene Deutung.

\section{Agrypon melanomerum FöRSTER, 1860, S. 151}

1 Ex.: Lectotypus (hiermit festgelegt): „18|121“, „Frst“, „melanomerum Foerst.“ (ZMHB).

1 Ex.: Paralectotypus: „180|92“, „Aachen Mai“, „Frst“ (ZMHB).

Locus typicus: Aachen.

Gültiger Name: Camposcopus nigricornis (WeSMAeL, 1849) (= Agrypon melanomerum FörSTER, syn. n.).

Der Nervellus ist, wie für die Art typisch, sehr weit unten nur ganz leicht gebrochen und die Discoidella sehr undeutlich. Förster hat das übersehen, so dass er die Art bei Agrypon einreihte.

\section{Agrypon elegantulum FörSTER, 1860, S. 152}

1 ㅇ: Lectotypus (hiermit festgelegt): „17|884“, „Frst“, „elegantulum Foerst.“ (ZMHB).

2 Ex.: Paralectotypen: „17|884“ bzw. „18|825“, „Aachen Mai“ (ZMHB).

Locus typicus: Aachen.

Gültiger Name: Agrypon flaveolatum (Gravenhorst, 1807) (= A. elegantulum Förster, syn. n.). 


\section{Agrypon aggressorium FöRSTER, 1860, S. 152}

$10^{\star}$ : Lectotypus (hiermit festgelegt): „Meig. Tab. XXV.14“, „Anomalon agressorium“(!) (FöRSTERS Handschrift) (ZMHB).

Gültiger Name: Agrypon flaveolatum (Gravenhorst, 1807) (= A. aggressorium Förster, syn. n.).

\section{Agrypon brachypterum FöRSTER, 1860, S. 152}

1 온 Lectotypus (hiermit festgelegt): „17|876“, „Frst“, „brachypterum Foerst“ (ZMHB).

1 o $^{\star}$ : Paralectotypus: „17|886“, „Frst“ (ZMHB).

Gültiger Name: Agrypon clandestinum (Gravenhorst, 1829) (= A. brachypterum Förster, syn. n.).

Der Nervellus ist beim Lectotypus fast ungebrochen, aber sonst sind die typischen Merkmale von $A$. clandestinum vorhanden. Der Paralectotypus ist nicht konspezifisch mit dem Lectotypus, sondern ein kleines Exemplar von A. flexorium (Thunberg, 1822) mit ungebrochenem Nervellus und etwas atypischem rötlichgelben Schläfenfleck.

\section{Agrypon furtivum FörSTER, 1860, S. 152}

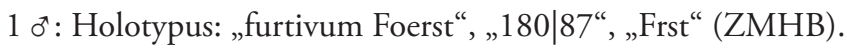

Gültiger Name: Agrypon anomelas (Gravenhorst, 1829) (= A. furtivum Förster, syn. n.).

\section{Agrypon macrurum FöRSTER, 1860, S. 152}

Typen verschollen.

Nomen dubium.

Da die Bohrerlänge nach FörsTer ein Drittel der Metasoma-Länge betragen haben soll, handelt es sich wahrscheinlich um eine Trichomma-Art, wobei der Autor die Augenbehaarung übersehen haben könnte.

\section{Agrypon cognatum FöRSTER, 1860, S. 152}

1 ơ: Holotypus: „180|70“, „Frst“, „,ognatum Foerst“ (ZMHB).

Gültiger Name: Agrypon varitarsum (Wesmael, 1849) (= A. cognatum FöRster, syn. n.).

\section{Agrypon confusum FöRSTER, 1860, S. 152}

$10^{\star}$ : Lectotypus (hiermit festgelegt): „17|828“, „Frst“, „confusum Foerst“ (ZMHB).

Gültiger Name: Agrypon flaveolatum (Gravenhorst, 1807) (= A. confusum Förster, syn. n.).

\section{Agrypon rubricatum FöRSTER, 1860, S. 153}

1 ㅇ: Lectotypus (hiermit festgelegt) „17|826“, „Frst“, „rubricatum Foerst“ (ZMHB).

1 Ex.: Paralectotypus: „17|881“, „Frst“, rubricatum Foerst“ (ZMHB).

1 ㅇ : „17/885“, „Frst“, „var. a” (ZMHB).

Gültiger Name: Agrypon flaveolatum (Gravenhorst, 1807) (= A. rubricatum Förster, syn. n.). 
Försters Art ist die rote Form von A. flaveolatum. Die drei vorhandenen Exemplare variieren in der Rotfärbung des Thorax, wie das bei dieser Form häufig vorkommt (siehe auch SchneE 1989). Atanasov (1982) hat ein von Kriechbaumer etikettiertes Tier („leg. 4.5.65“ !) aus der Zoologischen Staatssammlung München irrtümlicherweise für einen Typus von Atrometus rubricatus FöRSTER, 1878 („Lectotypus rubricatum“) gehalten und deshalb diesen Namen als Synonym von A. flaveolatum angesehen.

\section{Agrypon serpentinum FörSTER, 1860, S. 153}

1 ㅇ: Lectotypus (hiermit festgelegt): „Meigentab. XXV.17.“, „17|882“, „Frst“, „serpentinum Foerst" (ZMHB).

1 : Paralectotypus: „17|889“, „Aachen August“, „Frst“ (ZMHB).

Locus typicus: Aachen.

Gültige Name: Agrypon batis (Ratzeburg, 1852) (= A. serpentinum Förster, syn. n.).

\section{Laphyctes mesozonus FöRSTER, 1878, S. 72}

Locus typicus: Berlin.

Typen verschollen.

Die Deutung erfolgte nach der Beschreibung Försters und nach den Holotypen von Anomalon longicorne (BRAUns 1895) sowie von Barylypa frisiaca (HABERMEHL 1922a):

Anomalon longicorne Brauns 1895, S. 46

1 ㅇ : „Holotypus „Hym. Typ. Nr. 137“, „Bpest“ „40“, „Phalera bucephala“ (Wirtspuppe unter Holotypus) (HNHM)

Locus typicus: Budapest.

Barylypa frisiaca HABERMEHL 1922, S. 348-349

1 : Holotypus: „Neugraben 17.Sept. 16“, Barylypa frisiaca ㅇ Haberm. Prof. Habermehl det.“, „Typus" (SMF)

Locus typicus: Neugraben (bei Hamburg).

Gültiger Name: Barylypa mesozona (FöRster) (= Anomalon longicorne Brauns, 1895, syn. n., = Barylypa frisiaca HaBERMEHL, 1922, syn. nov.).

Die ausführliche Beschreibung trifft auch voll auf eine Serie von mir aus Phalera bucephala (Linnaeus) (Notodontidae) gezüchteter Barylypa-Exemplare zu, die bisher als B. longicornis galten. Der Holotypus von Anomalon longicorne stammt ebenfalls aus dieser Wirtsart. HABERMEHL (1922b) hat die Synonymie von longicorne und frisiaca nicht erkannt, denn er zitiert beide Namen auf derselben Seite für verschiedene Taxa. Von Yu \& Horstmann (1997) werden B. mesozona und $B$. frisiaca als Synonyme von $B$. delictor (ThunberG) angesehen. Das ist aber nicht korrekt, weil $B$. mesozona zu der Artengruppe mit fast körperlangen Fühlern gehört, während $B$. delictor die Gruppe mit kurzen Fühlern repräsentiert.

Die Art ist in Mitteleuropa offenbar selten und scheint nur während Massenvermehrungen der Wirts-Notodontide lokal häufiger vorzukommen.

B. mesozona ist bisher aus Deutschland, Ungarn, Russland (Meyer 1934, Vorontsov \& Marushina 1984) und Polen (KazmierczaK 1993) bekannt. 


\section{Laphyctes insidiator FörSTER, 1878, S. 73}

Holotypus im ZMHB nicht mehr vorhanden, lediglich Nadel mit folgenden Etiketten: „18|116“, „Aachen Juli“, „Insidiator Frst o“ “, „Holotype Laphyctes insidiator Först. Tow'64“

1 요 „18|115“, „Rheinprovinz“, „Frst“, „det. Sarntheinia insidiator by Foerster Tow'64“ (ZMHB).

Locus typicus: Aachen.

Gültiger Name: Barylypa propugnator (FörsTER, 1855) (= Laphyctes insidiator FöRSTER syn. n.).

Townes hat den Holotypus noch gesehen und das vorhandene Exemplar als von Förster determiniert bezeichnet. Deshalb kann die Konspezifität beider Tiere als gesichert gelten. Das vorhandene stark beschädigte Exemplar entspricht der Beschreibung. Es ist allerdings kein Syntypus, da FörsTer es nicht erwähnt. Eine völlige Übereinstimmung ergab sich auch durch den direkten Vergleich mit dem Holotypus von Anomalon propugnator.

\section{Atrometus insignis FöRSTER, 1878, S. 77}

$10^{\star}$ : Lectotypus (von Townes et al. 1965 festgelegt): „180|66“, „Frst“, „Montpellier“, „insignis Foerst" (ZMHB).

1 ơ: Paralectotypus (von Townes 1965 designiert): „180|65“, „Frst“ (ZMHB).

1 ㅇ: Paralectotypus (von Townes 1965 designiert): „180|96“, „Frst“, „Montpellier“ (ZMHB).

Locus typicus: Montpellier (Frankreich).

Gültiger Name: Atrometus insignis FörsTER, 1878.

\section{Atrometus rubricatus FöRSTER, 1878, S. 79}

1 ơ: Holotypus: „Grenada“, „24600“, „Type“ (ZMHB).

Locus typicus: Granada (Spanien).

Gültiger Name: Atrometus insignis FörSTER, 1878 (= Atrometus rubricatus FörsTer, syn. nov.).

Townes (1971) vermutete die hier bekräftigte Synonymie. Im Gegensatz dazu stellten AtANAsov (1982) und Ju \& Horstmann (1997) das Taxon zu Agrypon flaveolatum (Gravenhorst). (Siehe Erläuterung zu Agrypon rubricatum FörsTER).

\section{Zusammenstellung der nomenklatorischen Ergebnisse}

Erigorgus foersteri (MOCSÁRY, 1897), nom. n. pro Anomalon brevicorne FöRSTER, 1855 (= jüngeres Homonym von Anomalon brevicorne Gravenhorst, 1829)

= Anomalon försteri Dalla Torre, 1900, jüngeres Homonym

=E. melanops auct.

Barylypa rufa (HoLMgren, 1857)

= ? Anomalon vicinum Förster, 1855

Barylypa propugnator (FöRSTER, 1855)

= Laphyctes insidiator FöRSTER, 1878, syn. nov.

= Anomalon carinatum BrIsCHKE, 1880, syn. nov.

= Anomalon rufum Habermehl, 1920, syn. nov. 
(= jüngeres primäres Homonym von Anomalon rufum Holmgren, 1857)

Barylypa mesozona (FöRSTER, 1878)

= Anomalon longicorne Brauns, 1895, syn. nov.

= Barylypa frisiaca HABERMEHL, 1922, syn. nov.

Agrypon flexorium (Thunberg, 1822)

= Agrypon subclavatum Förster, 1860, syn. nov.

Agrypon clandestinum (Gravenhorst, 1829)

= Agrypon clandestinum FöRSTER, 1860, syn. nov.

(= jüngeres sekundäres Homonym von Anomalon clandestinum GravenHorst, 1829)

= Agrypon brachypterum FörSTER, 1860, syn. nov.

Agrypon flaveolatum (GravenHorst, 1807)

= Agrypon elegantulum Förster, 1860, syn. nov.

= Agrypon aggressorium FöRSTER, 1860, syn nov.

= Agrypon confusum FörsTER, 1860, syn. nov.

= Agrypon rubricatum FöRSTER, 1860, syn. nov.

Agrypon anomelas (Gravenhorst, 1829)

= Agrypon furtivum Förster, 1860, syn. nov.

Agrypon macrurum Förster, 1860, nom. dub.

Agrypon varitarsum (WeSMAeL, 1849)

= Agrypon cognatum FöRSTER, 1860, syn. nov.

Camposcopus nigricornis (WeSMAeL, 1849)

= Agrypon melanomerum FöRSTER, 1860, syn. nov.

Atrometus insignis Förster, 1878

= Atrometus rubricatus FöRSTER, 1878, syn. nov.

\section{Übersicht der übrigen Anomaloninae in Försters Sammlung}

Außer den meisten Typen ist auch der größte Teil der übrigen Anomaloninen Försters erhalten geblieben. Sie bilden ein reichhaltiges Material überwiegend aus Nordrhein-Westfalen von historisch-faunistischem Wert, dessen Publikation der Autor aber unterlassen hat. Das ist insofern positiv zu bewerten, da es zahlreiche in litteris-Arten enthält, die zumeist Synonyme geworden wären. Allerdings befanden sich in der FörsTER-Sammlung auch zehn wirklich neue Arten, die im Laufe der Zeit von späteren Autoren beschrieben worden sind. In der folgenden Zusammenstellung wird auf die Nennung der vielen unpublizierten Namen sowie der Etikettierung, die nicht zu deuten ist, verzichtet. Auch andere Fehldeutungen Försters werden nicht erwähnt.

Mit „Thom“ etikettierte Tiere hat Förster offenbar von Thomson aus Schweden erhalten. Fehlende Fundortangaben sind im Folgenden mit „o. F.“ gekennzeichnet. 


\section{Agrypon anxium (WeSMAEL, 1849)}

2 우 오 : „Montjoie Juni 67“, „Aachen 75“

Die beiden Exemplare repräsentieren jeweils unpublizierte Erstnachweise dieser aus Belgien beschriebenen Art für Frankreich und Deutschland. Der erste publizierte Nachweis für das damalige Deutschland stammt von BRISCHKE (1880).

\section{Agrypon canaliculatum (RATZEBURG, 1844)}

3 Ex.: „Tschek 1872 Piesting“

Tscheкs Exemplare stellen den ersten Fund dieses weit verbreiteten Parasitoiden von Gespinstmotten (Yponomeuta spp.; Yponomeutidae) für Österreich dar.

\section{Agrypon clandestinum (GraVENHORST, 1829)}

2 ㅇ ㅇ 1 Ex.: „Aachen Aug.74“, „Aachen Sept.“

Die überwiegend im Spätsommer auftretende, holarktisch vorkommende Art parasitiert kleine Geometriden.

\section{Agrypon delarvatum (GRAVENHORST, 1829)}

1 우 1 o $^{\star}, 2$ Ex.: „Tschek 1872 Piesting“, „Rh. Pr. August“ (= Rheinprovinz) und o. F.

Die Art gehört zu den wenigen Anomaloninen, die bei einigen Tagfalterarten aus den Familien Hesperiidae und Nymphalidae parasitieren. Ihr Verbreitungsgebiet erstreckt sich bis zur Ostpaläarktis.

\section{Agrypon flaveolatum (GRAVENHORST, 1807)}

20 우 우 đ $^{\star}$ : „Aachen Juni 75“, „Aachen Juni“ „Hannover“, „Kiel/Mai“, „Schweiz“ und o. F. Im Frühjahr ist $A$. flaveolatum in Europa nördlich der Alpen die häufigste Art der Unterfamilie. Die Larven leben vor allem in einer Reihe von Geometridenarten.

\section{Agrypon flexorioides SCHNEE, 1989}

5 우 우, $10^{\star}, 1$ Ex.: „Rh. Pr. 9.73“ und o. F.

Es handelt sich hier um die bisher nördlichsten Funde der überwiegend südlich (bis Rumänien und Frankreich) verbreiteten Art. In Deutschland ist sie ansonsten in Bayern und Thüringen nachgewiesen. Die Wirte sind bisher noch nicht bekannt.

\section{Agrypon flexorium (ThunberG, 1822)}

18 우 우 ơ ơ : „Aachen Juni 74-76“, „Aachen Mai 75-76“, „Aachen Aug. 75“, „Saisser Alp“ und o. F.

Die sehr polyphage Art hat zwei Generationen im Jahr, wie auch die Fangdaten aus Aachen andeuten. Sie ist sowohl morphologisch als auch farblich sehr variabel, hat ein breites Wirtsspektrum aus einigen Lepidopteren-Familien und gehört zu den häufigsten Anomaloninen in Europa. Ihr Areal erstreckt sich bis zur Ostpaläarktis. 
Agrypon gracilipes (CURTIS, 1839)

2 우 우 4 $40^{\star} 0^{\star}, 5$ Ex.: „Aachen Juni“, „Aachen Sept.“, „7.6.62 Ld.“ und o. F.

Försters Exemplare stellen den ersten Fund der seltenen Art für Deutschland dar. Es existieren bisher Wirtsnachweise aus einer Depressaria-Art (Depressariidae) in Österreich und aus dem Maiszünsler [Ostrinia nubilalis (HüBNER)] (Pyralidae) in Spanien.

\section{Agrypon polyxenae (SZÉPLIGETI, 1899)}

7 Ex.: „Deutschland“, „Wien“ und o. F.

In Deutschland in den jetzigen Grenzen kommt diese Art ebenso wenig vor wie ihre Wirte aus den Papilioniden-Gattungen Zerynthia und Archon. Sie ist A. flexorium sehr ähnlich, lässt sich aber durch die hinter den Augen erweiterten Schläfen, den stark verbreiterten Postpetiolus und das gedrungene Propodeum sowie offensichtlich auch durch die oligophage Lebensweise bei Papilioniden unterscheiden.

\section{Agrypon rugifer (ТномsоN, 1894)}

1 Ex.: „Deutschland“.

Die Art ist in Mitteleuropa selten, im Norden Europas dagegen häufiger. Sie wurde in der Vergangenheit oft mit Agrypon anxium (Wesmael, 1849), A. batis (Ratzeburg, 1852) und auch Perisphincter brevicollis (Wesmael, 1849) verwechselt. Als Wirt ist bisher nur die kleine Noctuide Hypena crassalis (FABRICIUS) ermittelt worden.

\section{Anomalon cruentatum (GEOFFroy, 1785)}

8 우 ㅇ, 7 ○ o : „Rheinprovinz“, „Aachen“, „Schweden“, „Thom.“, “Italia“ und o. F.

In der nördlichen Westpaläarktis ist die weit verbreitete Art ziemlich selten, im Mittelmeerraum dagegen durchaus häufig. Als einzige Art unter den europäischen Anomaloninen parasitiert sie nicht bei Lepidopteren, sondern soweit bisher bekannt, bei Tenebrioniden-Larven.

\section{Anomalon sp.}

2 우 우, $10^{\star}:$, „Aegypt.“ und o. F.

Es handelt sich hier möglicherweise um eine noch unbeschriebene Art.

\section{Aphanistes gliscens (HARTIG, 1838)}

3 우, 7 o $^{\star}$, 2 Ex.: „Aachen Juni“, „Aachen August“, „Aachen 75“, „Chur“ (Schweiz), „Köln“, "Thom".

Insbesondere in europäischen Kiefernwäldern ist die bei der Forleule [Panolis flammea (Denis \& SCHIfFERmüLler)] und einigen anderen Noctuiden parasitierende Art nicht selten.

\section{Aphanistes klugii (HARTIG, 1838)}

2 우: "Hannover".

Diese größte Aphanistes-Art ist in der westlichen Paläarktis ein regelmäßiger Parasitoid des Kiefernschwärmers [Hyloicus pinastri (LinnaEus)] (Sphingidae). 


\section{Aphanistes ruficornis (GraVENHORST, 1829)}

\section{Ex.: „Hanover“}

Als Wirte der aus vielen Ländern Europas, aber auch aus Korea nachgewiesenen Art fungieren einige Notodontidae und Noctuidae.

\section{Barylypa pallida (GRAVENHORST, 1829)}

$2 o^{x} o^{x}$ : "Aachen" und o. F.

In Europa nördlich der Alpen kommt die Art nur sehr selten vor. Ihre Wirte sind bisher unbekannt.

\section{Barylypa propugnator (FÖRSTER, 1855)}

1 Ex.: „Niederw. 8.74“

Die Art ist in Europa weit verbreitet und parasitiert bei einigen Noctuiden-Arten, vor allem bei Cucullia spp.

\section{Barylypa uniguttata (GRAVENHORST, 1829)}

5 Ex.: "Köln", "Aachen Juni" und o. F.

Die normale Flugzeit der Art liegt in Mitteleuropa zwischen Ende März und Mitte Mai.

Das Verbreitungsgebiet erstreckt sich auf ganz Europa. Die Wirtslepidopteren sind noch nicht bekannt.

\section{Erigorgus annulitarsis (ТномsоN, 1892)}

$10^{*}:$ "Ungarn"

Ihre Hauptverbreitung hat die Art im Mittelmeerraum. Sie kommt aber auch im übrigen Europa vor. Als Wirt konnte bisher die Noctuide Xestia xanthographa (Denis \& SchiffermüLler) festgestellt werden (SCHNEE 1991).

\section{Erigorgus apollinis KRIECHBAUMER, 1900}

$1 \sigma^{\top}$ : „apollinis Kriechb. aus Doritis apollinis“ (Försters Handschrift), „Berchtesgaden Anomalon apollinis ơ mihi e pupa Doritis apollinis" (Kriechbaumers Handschrift)

$10^{\star}:$ : Berchtesgaden“

Das 1. Exemplar ist ein Paralectotypus des von Kriechbaumer beschriebenen Parasitoiden von Parnassius apollo (Linnaeus). Der Lectotypus befindet sich in der Zoologischen Staatssammlung München.

\section{Erigorgus cerinops (GRAVENHORST, 1807)}

6 ơ $^{\star} o^{\star}:$, „Köln“, „Thom.“, „Aachen“ und o. F.

Im Gegensatz zu allen anderen europäischen Erigorgus-Arten, die im Frühjahr fliegen und monovoltin sind, ist E. cerinops eine bivoltine Art, deren Flugzeit sich von Juni bis Oktober erstreckt. Wichtigste Wirte sind die Erdraupen der Wintersaateule [Agrotis segetum (DeNIs \& SChiffermüller)] (Noctuidae). 


\section{Erigorgus femorator (AUBERT, 1960)}

$10^{3}:$ o. F.

Das nördlichste Vorkommen der im mediterranen Raum häufigen, beim Pinienprozessionsspinner [Thaumetopoea pithyocampa (DenIs \& SCHIFFERMüLLER)] (Notodontidae) parasitierenden Art ist aus der Südschweiz bekannt.

\section{Erigorgus fibulator (GRAVENHORST, 1829)}

2 Ex.: „Tschek 1872 Piesting“, 1 ơ $^{\star}$ o. F.

Wirtsnachweise existieren für die Art, deren Areal sich von Europa bis in die Ostpaläarktis erstreckt, noch nicht.

\section{Erigorgus latro (SCHRANK, 1781)}

3 ㅇ $20^{x} o^{\star}:$ o. F.

Nach bisheriger Kenntnis parasitiert E. latro monophag in den Raupen von Diloba caeruleocephala (Linnaeus) (Noctuidae). In Heckenhabitaten, in denen der Wirt regelmäßig vorkommt, kann sie lokal durchaus häufig sein.

\section{Erigorgus melanobatus (GRAVENHORST, 1829)}

1 오 : „Chur“

Zumindest in Europa ist die transpaläarktisch verbreitete Art sehr selten. Wirte sind noch nicht bekannt.

\section{Erigorgus melanops (FöRSTER, 1855)}

$9 \sigma^{\star} \sigma^{\star}:$, „Montjoie“, „Rheinprovinz“

Diese Art ist ebenso wie die folgende in Europa weit verbreitet und parasitiert vor allem die Raupen verschiedener Noctuiden (ScHNEE 1991), aber nach Zuchtergebnissen von SHAw (unpubliziert) auch einiger Nymphaliden.

\section{Erigorgus procerus (GRAVENHORST, 1829)}

1 o 1 o : „Aachen“ und o. F.

Als Wirte der bis in die Ostpaläarktis vorkommenden Art sind einige Noctuidenarten ermittelt worden (SCHNEE 1991).

\section{Erigorgus varicornis (ТномsоN, 1894)}

1 요 :Aachen”, 1 요 : o. F.

E. varicornis ist in Nordeuropa häufiger als im Süden. Es liegen bisher keine Wirtsnachweise vor.

\section{Erigorgus villosus (GRAVENHORST, 1829)}

1 ㅇ: „Berchtesgaden“, 1 i : „German mar.“ 
Das Vorkommen erstreckt sich von Finnland bis Griechenland. Es existieren aber nur sehr sporadische Nachweise. Die Angabe von Laboulbène (1877), wonach Giraud die Art aus Lemonia dumi (Linnaeus) (Lemoniidae) gezüchtet haben soll, konnte nicht überprüft werden.

Das Material ist in Paris offenbar nicht mehr vorhanden.

\section{Habronyx heros WeSMAEL, 1849}

3 Ex.: „Rh. Pr. 9.73 Wesm.“, „Aachen“ und o. F.

Auch heute kommt diese größte westpaläarktische Anomalonine noch in Südeuropa vor. Dagegen scheint sie in Mitteleuropa ausgestorben zu sein.

\section{Heteropelma amictum (FABRICIUS, 1775)}

5 우 우 $10^{\star}:$, „München”, „Rossia”, „Aachen Mai 75” und o. F.

Die nicht seltene Art ist transpaläarktisch und darüber hinaus auch in der Orientalis verbreitet. Nach eigenen Zuchterfahrungen scheint in Europa die Arctiide Panaxia dominula (Linnaeus) der wichtigste Wirt zu sein.

\section{Heteropelma grossator SHESTAKOV, 1923}

1 우: o. F.

Im Gegensatz zur vorigen ist diese Art außerordentlich selten. Nachweise gibt es bisher aus Russland, Österreich, Ungarn und der Schweiz. Als Wirt wurde von HeInrich (1953) die Nymphalide Lasiommata maera (LinNaEus) ermittelt.

\section{Heteropelma megarthrum (RATZEBURG, 1848)}

8 우 ㅇ $40^{\star} 0^{\star}$ : „Aachen Juli 74“, „Aachen Juli 75“, „Aachen Juni“, „Aachen Sept.“, „Tegernsee“, „Pommern“"

Das aus Pommern stammende Männchen ist von FörsTer als „megarthrum Rtzb. calcator Wesm." etikettiert worden. Er hat also die von späteren Autoren übersehene Synonymie erkannt. H. megarthrum ist eine sehr häufige Art und ein wichtiger Parasitoid des Forstschädlings Bupalus piniarius (Linnaeus) (Geometridae).

\section{Parania geniculata (HoLMgren, 1857)}

1 Ex.: o. F.

Die kleinste europäische Anomalonine kommt auch in der Nearktis vor und parasitiert vorzugsweise bei Tortriciden.

\section{Therion circumflexum (LINNAEUs, 1758)}

5 우 우 4 o $^{\star} 0^{\star}$ : „Schweiz”, „Schweiz 76”, „Aachen Juni 75”, „Aachen Juli 74”

Die holarktische Art ist in Mitteleuropa vor allem in Kiefernwäldern häufig. Als Wirte sind mehrere Noctuidenarten bekannt. 


\section{Therion giganteum (GRAVENHORST, 1829)}

2 우 우 $10^{\star}:$ : „Aachen Sept.“, „Hanover“

Bei Massenvermehrungen des wichtigsten Wirtes Dendrolimus pini (Linnaeus) (Lasiocampidae) ist die Art in Europa nicht selten. Ihr Verbreitungsgebiet reicht bis zur Ostpaläarktis.

\section{Therion tarsatum (SHESTAKOV, 1923)}

1 : : „Ungarn...10.9...”

Es handelt sich hierbei um den einzigen bisher bekannten Fund der sehr seltenen Art aus Ungarn. Wirtsangaben fehlen bisher.

\section{Trichomma enecator (RossI, 1790)}

1 o 7 Ex.: „Rheinprov.”, „Aachen Mai 76”, „Aachen August 74”, „Aachen Sept. 74“, „Aachen Juni $76^{\prime \prime}$

Dieser Parasitoid vor allem des Apfelwicklers [Cydia pomonella (Linnaeus)], aber auch anderer Tortriciden, ist in Europa nicht selten.

\section{Trichomma fulvidens WeSMAEL, 1849}

1 o 3 Ex.: „Aachen April 74,75,76“ und o. F.

T. fulvidens gehört zu den im Frühjahr fliegenden häufigeren Anomaloninen. Sie parasitiert bei Noctuiden (Schnee 1991). Ihr Areal reicht von Europa bis Japan und Korea.

\section{Trichomma intermedium KRIEGER, 1904}

1 o 3 Ex.: „Aachen“ und o. F.

Die selten gefundene Art ist lediglich aus einigen europäischen Ländern bekannt. Sie parasitiert nach Haeselbarth (1979) bei der Geometride Ectropis crepuscularia (Denis \& SchifferMÜLLER).

\section{Trichomma occisor HABERMEHL, 1909}

2Ex.: o. F.

Wirte dieser ebenfalls nicht häufigen Art sind aus Europa bisher nicht bekannt. In Korea wurde sie aus einigen Pyraliden gezüchtet (Lee \& Cha 2000).

\section{Danksagung}

Den Herren Dr. F. Косн (Berlin) und Dr. J. PApp (Budapest) gebührt mein Dank für die Arbeitsmöglichkeit in den von ihnen betreuten Sammlungen sowie den Herren E. Diller (München) und Dr. J.-P. Kopelke für die Ausleihe einiger Anomaloninae. Herrn Dr. K. Horstmann bin ich für die freundliche leihweise Überlassung der in seinem Besitz befindlichen Kopien der Notizbücher Försters dankbar. 


\section{Literatur}

Atanasov, A. Z. 1975: Novye predstaviteli i opredelitelnaya tablitsa palearkticheskikh vidov roda Erigorgus (Hymenoptera, Ichneumonidae). - Zoologicheski zhurnal, Moskva 54: 1480-1487.

AtanAsov, A. Z. 1982: Ob obeme i nomenklature nekotorykh rodov nodsemeystva Anomaloninae (Hymenoptera, Ichneumonidae). - Acta Zoologica Bulgarica, Sofia 19: 75-77.

Bachmaier, F. 1979: Das in der Zoologischen Staatssammlung München aufbewahrte Typenmaterial der Gattung Dusona Cameron 1900 (= Campoplex auct.) und sein Erhaltungszustand (Hymenoptera, Ichneumonidae, Porizontinae). - Spixiana, München 2: 69-93.

BRAUns, S. 1895: Descriptiones speciorum novarum Ichneumonidarum e fauna Hungarica. - Természetrajzi Füzetek, Budapest 18: 42-49.

Brischke, V. G. 1880: Die Ichneumoniden der Provinz West- und Ostpreußen. I. Fortsetzung. - Schriften der naturforschenden Gesellschaft Danzig (N. F.), Danzig 4: 108-210.

Diller, E. 1993: Briefliche Mitteilung.

FöRSTER, A. 1855: Die 2te Centurie neuer Hymenopteren. - Verhandlungen des Naturhistorischen Vereins der Preussischen Rheinlande und Westfalens, Bonn 12: 226-258.

FöRSTER, A. 1860: Eine Centurie neuer Hymenopteren. - Verhandlungen des Naturhistorischen Vereins der Preussischen Rheinlande und Westfalens, Bonn 17: 93-153.

Förster, A. 1869: Synopsis der Familien und Gattungen der Ichneumonen. - Verhandlungen des Naturhistorischen Vereins der Preussischen Rheinlande und Westfalens, Bonn 25: 135-221.

Förster, A. 1878: Synopsis der Familien und Gattungen der Ichneumonen. - Verhandlungen des Naturhistorischen Vereins der Preussischen Rheinlande und Westfalens, Bonn 35: 42-82.

Habermehl, H. 1920: Neue und wenig bekannte paläarktische Ichneumoniden (Hym.). - Deutsche entomologische Zeitschrift, Berlin 1920: 315-330.

Habermehl, H. 1922a: Neue und wenig bekannte paläarktische Ichneumoniden (Hym.). - Deutsche entomologische Zeitschrift, Berlin 1922: 348-359.

Habermehl, H. 1922b: Beiträge zur Kenntnis der palaearktischen Ichneumonidenfauna. - Konowia, Wien 1: 77-86.

Haeselbarth, E. 1979: Zur Parasitierung der Puppen von Forleule (Panolis flammea (L.)), Kiefernspanner (Bupalus piniarius (L.)) und Heidelbeerspanner (Boarmia bistortata (GoEzE)) in bayerischen Kiefernwäldern. Teil 1. - Zeitschrift für Angewandte Entomologie, Berlin 87: 186-202.

Heinrich, G. 1953: Ichneumoniden der Steiermark (Hym.). - Bonner Zoologische Beiträge, Bonn 4: 147-185.

Horstmann, K. 1990: Revision einiger Typen der von Otтo Schmiedeknecht beschriebenen paläarktischen Ichneumonidae (Hymenoptera). - Beiträge zur Entomologie, Berlin 40: 31-61.

Internationale Kommission für Zoologische Nomenklatur (ICZN) 2000: Internationale Regeln für die Zoologische Nomenklatur.- 4. Auflage - Offizieller deutscher Text. - Goecke \& Evers, Keltern-Weiler: 232 S.

Kazmierczak, T. 1993: Ichneumonidae of selected regions of southern Poland. - Acta Zoologica Cracoviensia, Kraków 36: 77-120.

Kiss Zilah, A. von 1929: Dritter Beitrag zur Kenntnis der ungarischen und siebenbürgischen Ichneumoniden-(Schlupfwespen-)fauna. - Verhandlungen und Mitteilungen des Siebenbürgischen Vereins für Naturwissenschaften in Hermannstadt, Hermannstadt 79/80: 89-144.

Kriechbaumer, J. 1880: Gezogene Schlupfwespen aus Dalmatien. - Entomologische Nachrichten, München 6: 73-75.

Laboulbène, A. 1877: Liste des éclosions d'insectes observées par le Dr. Joseph-Etienne Giraud, membre honoraire. - Annales de la Societé Entomologique de France, Paris 5 (7): 397-436.

LeE, J. W. \& Cha, J. Y. 2000: Illustrated Catalogue of Ichneumonidae in Korea (Hymenoptera). - Insects of Korea. - Korea research institute of bioscience and biotechnology \& center for insect systematic, Korea, 275 S., 297 Fig. 
Meyer, N. F. 1934: Schlupfwespen die in Russland in den letzten Jahren aus Schädlingen gezogen sind. - Zeitschrift für angewandte Entomologie, Berlin 20: 611-618.

MocsárY, A. 1879: Jellemzö adatok Budapest környékének hártyaröpü fanájához. Data characteristica ad faunam hymenopterologicam regionis Budapestinensis. - In: Budapest és környéke természetrajzi orvosi és közmivlödési leirása. Topographia medicina et physica regionis Budapestinensis 1: 364-368.

MocsárY, A. 1897: Ordo Hymenoptera. - In: A Magyar birodalom állatvilága. Fauna regni Hungariae. III. Arthropoda. (Ichneumonidae S. 33-57), Budapest.

Mocsáry, A. 1898: Magyarország Hymeopterái. Ungarns Hymenopteren. - Természetrajzi Füzetek, Budapest 21: 153-163.

Perkins, J. F. 1962: On the type species of Förster's genera (Hymenoptera: Ichneumonidae). - Bulletin of the British Museum (Natural History), London 11: 385-483.

Schmiedeknecht, O. 1902: Die Ichneumonidentribus der Anomalinen. (Hym.). Eine Übersicht sämmtlicher Gattungen sowie der paläarktischen Arten. - Zeitschrift für systematische Hymenopterologie und Dipterologie, Teschendorf 2: 356-368.

Schmiedeknecht, O. 1903: Die Ichneumonidentribus der Anomalinen. (Hym.). Eine Übersicht sämmtlicher Gattungen sowie der paläarktischen Arten. - Zeitschrift für systematische Hymenopterologie und Dipterologie, Teschendorf 3: 1-8, 73-80, 171-176.

Schmiedeknecht, O. 1935: Opuscula Ichneumonologica. Supplement-Band. Neubearbeitungen. - Fasc. XXIV. 4. Tribus Anomalonini, Blankenburg in Thüringen: 55-84.

Schmiedeknecht, O. 1936: Opuscula Ichneumonologica. Supplement-Band. Neubearbeitungen. - Fasc. XXV. 4. Tribus Anomalonini, Blankenburg in Thüringen: 85-140.

Schnee, H. 1989: Revision der von Gravenhorst beschriebenen und redeskribierten Anomaloninae mit Beschreibung zweier neuer Arten. - Deutsche Entomologische Zeitschrift, Berlin N. F. 36: 241-266.

Schnee, H. 1991: Zur Kenntnis der Phänologie und der Wirtsspektren einiger paläarktischer Anomaloninae (Hymenoptera, Ichneumonidae). - XII. Internationales Symposium zur Entomofaunistik Mitteleuropas Kiev, Vorträge: 80-85.

SzÉpligeti, G. 1899: Adatok a Magyarorszaági fürkészö darazsar ismeretéhez. Beiträge zur Kenntnis der ungarischen Ichneumoniden. - Természetrajzi Füzetek, Budapest 22: 213-246.

SzÉpligeti, G. 1905a: Hymenoptera. Ichneumonidae (Gruppe Ophionoidea), subfam. Pharsaliinaeporizontinae. - Genera Insectorum, Bruxelles 34: 1-68.

SzÉPLIGETI, G. 1905b: Übersicht der paläarktischen Ichneumoniden. I. Teil. - Annales Musei Nationalis Hungarici, Budapest 3: 508-540.

Townes, H. K. 1971: The Genera of Ichneumonidae, Part 4. - Memoirs of the American Entomological Institute, Ann Arbor 17: 372 S., 217 Fig.

Townes, H. K.; Momoi, S. \& Townes, M. 1965: A catalogue and reclassification of the eastern Palearctic Ichneumonidae. - Memoirs of the American Entomological Institute. No. 5, $661 \mathrm{~S}$.

Vorontsov, A. I. \& Marushina, N. G. 1973: Parasity lunki zerebristoi. - Voprosy zashchita lesa.- Nauchnye trudy Moskovskovo lesotekhnicheskovo instituta, Moskva 41: 86-94.

Yu, D. S. \& K. Horstmann 1997: A catalogue of World Ichneumonidae (Hymenoptera). - Memoirs of the American Entomological Institute 58 (1-2), VI \& 1558 S.

\section{Anschrift des Verfassers:}

Dipl.-Biol. Heinz Schnee

Birkenweg 18

04416 Markkleeberg

E-mail: Heinz.Schnee@web.de

\section{Subject editor:}

Dr. S. M. BLANK 